\title{
A Reliable Algorithm for Multi-Dimensional Mobile/ Immobile Advection-Dispersion Equation with Variable Order Fractional
}

Iman I. Gorial*

\author{
Department of Materials Engineering, University of Technology, Iraq; imanisho@yahoo.com
}

\begin{abstract}
Objectives: Presented a modified treatment of initial boundary value problems for multi-dimensional mobile/immobile advection-dispersion equation with variable order fractional (MDMADEVF). Methods: we applied mixed initial and boundary conditions together using variational iteration method (VIM) to get a new initial solution at every iteration. Findings: The simulation results show the proposed system accurate and the utilized algorithm simple and easy to implement. Application: numerical examples were provided to show that variational iteration method was computationally efficient. The results were presented in tables and figures using the MathCAD 12 and Matlab software package when it is needed.
\end{abstract}

Keywords: Caputo Derivatives, Fractional Mobile / Immobile Advection-Dispersion Model.

\section{Introduction}

Fractional Differential Equations (FDEs) display numerous phenomena in several fields such as engineering, finance and physics $\frac{1-3}{}$, viscoelasticity ${ }^{4}$, biology ${ }^{5}$, fluid mechanics and chemistry ${ }^{6-7}$. Therefore, a growing report by applying fractional calculus in signal processing, modeling, control, electromagnetism, physics, mechanics, bioengineering, medicine and in many other areas. Numerical and approximation techniques must be used due to most FDEs do not have exact analytic solutions.

Numerical treatment based upon finite difference methods for FDEswaspresented $\frac{8-10}{}$. While Finite element methods were introduced to obtain the numerical solutions of FDEs $s^{11-14}$. In addition, several spectral algorithms were designed for FDEs in previous reports $\frac{15-19}{}$. In these papers, fractional order derivative is constant fractional and not variable fractional order while is constant fractional.

Advection-dispersion equation was utilized to model many chemical, physical engineering and sci- ences ofearth ${ }^{20}$. Lately, researchers found that numerous dynamic processes displayed fractional order manner that might differ with space or time and showed that variable-order calculus was a natural candidate to supply an efficient mathematical framework for the depiction of complex dynamical problems $\frac{21-26}{2}$. In other papers different variable fractional operator definitions for solving variable FDEs were discussed ${ }^{27-33}$.

The numerical approximation of variable fractional order partial differential equations with is relatively new, and at an early stage of development. Additionally, the most developed methods today are finite difference methods for the numerical approximation of variable-order FDEs $\frac{34-37}{4}$.

In the last decade, there were exceptional consideration to suggest and develop spectral methods to solve FDEs with both variable-order and fixed-orderoperators $\frac{38-41}{}$.

$\mathrm{In}^{42}$ suggested spectral collocation method for solving $1 \mathrm{D}$ and $2 \mathrm{D}$ variable-order fractional nonlinear cable equations $s^{\underline{43}}$ developed an exponentially accurate frac-

*Author for correspondence 
tional spectral collocation method for solving linear/ nonlinear variable-order FDEs.

$\operatorname{In}^{44}$ proposed an accurate numerical algorithm for functional boundary value problems with variable-order fractional 145 suggested a numerical method for the time variable fractional order mobile-immobile advectiondispersion model (VFOMADM) ${ }^{46}$ developed a numerical simulation of time VFOMADM. The primary objective of this work was to present a (VIM) with modified treatment of initial boundary value problems. The secondary objective was to use this method to study numerically the (MDMADEVF) given by:

$$
\begin{aligned}
& \beta_{1} D_{\zeta} \Psi\left(w_{1}, w_{2}, \ldots, w_{n}, \zeta\right)+\beta_{2} D_{\zeta}^{\gamma\left(w_{1}, w_{2}, \ldots, w_{n}, \zeta\right)} \Psi\left(w_{1}, w_{2}, \ldots, w_{n}, \zeta\right) \\
& =-\beta_{3} \sum_{i=1}^{n} D_{w_{i}} \Psi\left(w_{1}, w_{2}, \ldots, w_{n}, \zeta\right)+\beta_{4} \sum_{i=1}^{n} D_{w_{i}}^{2} \Psi\left(w_{1}, w_{2}, \ldots, w_{n}, \zeta\right)+ \\
& Q\left(w_{1}, w_{2}, \ldots, w_{n}, \zeta\right), \quad i=1,2, \ldots, n
\end{aligned}
$$

subject to the initial condition (IC):

$$
\Psi\left(w_{1}, w_{2}, \ldots, w_{n}, 0\right)=f\left(w_{1}, w_{2}, \ldots, w_{n}\right), \quad 0 \leq w_{i} \leq 1
$$

And the boundary conditions (BC):

$$
\begin{aligned}
& \Psi\left(0, w_{2}, \ldots, w_{n}, \zeta\right)=k_{1}\left(w_{2}, w_{3}, \ldots, w_{n}, \zeta\right) \quad, 0 \leq \zeta \leq J \\
& \Psi\left(w_{1}, 0, \ldots, w_{n}, \zeta\right)=k_{2}\left(w_{1}, w_{3}, \ldots, w_{n}, \zeta\right) \\
& \bullet \\
& \text { • } \\
& \Psi\left(w_{1}, w_{2}, \ldots, w_{n-1}, 0, \zeta\right)=k_{n}\left(w_{1}, w_{2}, \ldots, w_{n-1}, \zeta\right) \\
& \Psi\left(1, w_{2}, \ldots, w_{n}, \zeta\right)=H_{1}\left(w_{2}, w_{3}, \ldots, w_{n}, \zeta\right) \\
& \Psi\left(w_{1}, 1, \ldots, w_{n}, \zeta\right)=H_{2}\left(w_{1}, w_{3}, \ldots, w_{n}, \zeta\right) \\
& \text { - } \\
& \text { • } \\
& \text { • } \\
& \Psi\left(w_{1}, w_{2}, \ldots, w_{n-1}, 1, \zeta\right)=H_{n}\left(w_{1}, w_{2}, \ldots, w_{n-1}, \zeta\right)
\end{aligned}
$$

Where $\beta_{1}, \beta_{2} \geq 0, \beta_{3}, \beta_{4}>0,0<\gamma \leq \gamma(w, \zeta) \leq \bar{\gamma} \leq 1$, and, $Q, f, k_{1}, k_{2}, \cdots, k_{n}$, and, $H_{1}, H_{2}, \ldots, H_{n}$ are known functions, $\mathrm{T}$ is given constantand $D^{a(x)}$ in our problem we define in terms of Caputo variable order fractional derivatives as defined by $\underline{\underline{47-48}}$

$$
D^{\alpha(w, \zeta)} f(w)=\frac{1}{\Gamma(m-\alpha(w, \zeta))} \int_{0}^{w} \frac{f^{(m)}(\zeta)}{(w-\zeta)^{\alpha(w, \zeta)-m+1}} d \zeta
$$

Where $m-1<\alpha(w)<m, m \in N, w>0$.

For the Caputo variable order derivative, we have:

$$
D_{L_{+}}^{\alpha(w)}(w-L)^{n}=\frac{\Gamma(n+1)}{\Gamma(n+1-\alpha(w))}(w-L)^{n-\alpha(w)}
$$

And

$$
D_{R-}^{\alpha(w)}(R-w)^{n}=\frac{\Gamma(n+1)}{\Gamma(n+1-\alpha(w))}(R-w)^{n-\alpha(w)}
$$

The paper is organized as follows. In section 2, modified treatment of initial boundary value. In section 3, VIM for solving (MDMADEVF). In section 4, numerical examples are solved using proposed method. Finally, we present conclusion about solution (MDMADEVF) in section 5 .

\section{Modified Treatment of Initial Boundary Value Problems}

Usually for selecting $\Psi_{0}$ the zeroth approximationused the initial values, but in this paper, we accredited a new technique to calculate the zero the approximation $\Psi_{0}^{*}$ by constructing a new initial solutions $\Psi_{n}{ }^{*}$ by mixed initial conditions with boundary conditions at every iteration as follows:

First, the initial solution can be written as:

$$
\Psi_{0}\left(w_{1}, w_{2}, \ldots, w_{n}, \zeta\right)=f_{0}\left(w_{1}, w_{2}, \ldots, w_{n}\right)+\zeta f_{1}\left(w_{1}, w_{2}, \ldots, w_{n}\right)
$$

Where

$$
\begin{aligned}
& \Psi\left(w_{1}, w_{2}, \ldots, w_{n}, 0\right)=f_{0}\left(w_{1}, w_{2}, \ldots, w_{n}\right), \\
& D_{\zeta} \Psi\left(w_{1}, w_{2}, \ldots, w_{n}, 0\right)=f_{1}\left(w_{1}, w_{2}, \ldots, w_{n}\right)
\end{aligned}
$$

Second, we constructed a new successive initial solution $\Psi_{n}^{*}$ atevery iteration by applying a new technique

$$
\begin{aligned}
\Psi_{n}^{*} & \left(w_{1}, w_{2}, \ldots, w_{n}, \zeta\right) \\
& =\Psi_{n}\left(w_{1}, w_{2}, \ldots, w_{n}, \zeta\right)+\left(1-w_{1}^{2}\right) \\
& {\left[k_{1}\left(w, w_{2}, \ldots, w_{n}, \zeta\right)-\Psi_{n}\left(0, w_{2}, \ldots, w_{n}, \zeta\right)\right]+} \\
& w_{1}^{2}\left[H_{1}\left(w_{1}, w_{2}, \ldots, w_{n}, \zeta\right)-\Psi_{n}\left(0, w_{2}, \ldots, w_{n}, \zeta\right)\right]+\ldots+ \\
& (1-w)\left[k_{n}\left(w_{1}, w_{2}, \ldots, w_{n}, \zeta\right)-\Psi_{n}\left(w_{1}, w_{2}, \ldots, w_{n}, \zeta\right)\right]+ \\
& w_{n}^{2}\left[H_{n}\left(w_{1}, w_{2}, \ldots, w_{n-1}, \zeta\right)-\Psi_{n}\left(w_{1}, w_{2}, \ldots, w_{n-1}, 1, \zeta\right)\right]
\end{aligned}
$$


Where

$$
\begin{gathered}
\Psi\left(0, w_{2}, \ldots, w_{n}, \zeta\right)=k_{1}\left(w_{2}, w_{3}, \ldots, w_{n}, \zeta\right) \\
\vdots \\
\Psi\left(w_{1}, w_{2}, \ldots, w_{n-1}, 0, \zeta\right)=k_{n}\left(w_{1}, w_{2}, \ldots, w_{n-1}, \zeta\right) \\
\Psi\left(1, w_{2}, \ldots, w_{n}, \zeta\right)=H_{1}\left(w_{2}, w_{3}, \ldots, w_{n}, \zeta\right) \\
\vdots \\
\Psi\left(w_{1}, w_{2}, \ldots, w_{n-1}, 1, \zeta\right)=H_{n}\left(w_{1}, w_{2}, \ldots, w_{n-1}, \zeta\right)
\end{gathered}
$$

\section{VIM for Solving MDMADEVF}

In this section, we will apply VIM for solving MDMADEVF. Consider the general nonlinear differential equation:

$$
L \Psi(w, \zeta)+N \Psi(w, \zeta)=g(w, \zeta)
$$

Where $L$ is a linear differential operator, $N$ is a nonlinear operator, and $g$ an inhomogeneous term. According to VIM:

$$
\Psi_{n+1}(w, \zeta)=\Psi_{n}(w, \zeta)+\int_{0}^{\zeta} \lambda\left(L \Psi_{n}(\tau)+N \Psi_{n}(\tau)-g(\tau)\right) d \tau
$$

And $\lambda$ is a Lagrange multiplier which can be identified optimally via the variational theory. The subscript $n$ indicates the $n$th approximation and $\tilde{\Psi}_{n}$ is considered as a restricted variation $\delta \tilde{\Psi}_{n}=0$.

To solve problem (1), for simplicity, according to the VIM and modified treatment of initial boundary value problems, we will derive functional correction as follow:

$$
\begin{aligned}
& \Psi_{n+1}(w, \zeta)=\Psi_{n}^{*}(w, \zeta)+\int_{0}^{\zeta} \lambda\left(\beta_{1} D_{\zeta} \Psi_{n}^{*}\left(w_{1}, w_{2}, \ldots, w_{n}, \tau\right)\right. \\
& +\beta_{2} D_{\zeta}^{\gamma\left(w_{1}, w_{2}, \ldots, w_{n}, \tau\right)}\left(\Psi_{n}^{*}\left(w_{1}, w_{2}, \ldots, w_{n}, \tau\right)\right. \\
& +\beta_{3} \sum_{i=1}^{n} D_{w_{i}} \Psi_{n}^{*}\left(w_{1}, w_{2}, \ldots, w_{n}, \tau\right)-\beta_{4} \sum_{i=1}^{n} D_{w_{i}}^{2} \Psi_{n}^{*}\left(w_{1}, w_{2}, \ldots, w_{n}, \tau\right) \\
& \left.-Q\left(w_{1}, w_{2}, \ldots, w_{n}, \tau\right)\right) d \tau, i=1,2, \ldots, n
\end{aligned}
$$

Where $\lambda=\frac{(-1)^{m}(\tau-\zeta)^{m-1}}{(m-1) !}$

\section{Test Problems}

In this section, we will present some examples to show efficiency and high accuracy of modified treatment of initial boundary value problems by VIM for MDMADEVF.

Example 1: Consider the following one-dimensionalequation $\frac{45-46 \text { : }}{2}$

$$
\begin{aligned}
& \beta_{1} D_{\zeta} \Psi\left(w_{1}, \zeta\right)+\beta_{2} D_{\zeta}^{1-0.5 e^{-m \zeta \zeta}} \Psi\left(w_{1}, \zeta\right)=-\beta_{3} D_{w_{1}} \Psi\left(w_{1}, \zeta\right) \\
& \quad+\beta_{4} D_{w_{1}}^{2} \Psi\left(w_{1}, \zeta\right)+Q\left(w_{1}, \zeta\right)
\end{aligned}
$$

Let $\beta_{1}=\beta_{2}=\beta_{3}=\beta_{4}=1$

And the source functions:

$$
\begin{aligned}
& Q\left(w_{1}, \zeta\right)=20 w_{1}(\zeta+1)\left(1-w_{1}\right)^{2}-20 w_{1}^{2}(\zeta+1)\left(1-w_{1}\right) \\
& \quad-2(10 \zeta+10)\left(1-w_{1}\right)^{2}-8 w_{1}(10 \zeta+10)\left(1-w_{1}\right) \\
& \quad+2(10 \zeta+10) w_{1}^{2}+10 w_{1}^{2}\left(1-w_{1}\right)^{2}\left(\frac{t^{5 e^{-w_{1}}}}{\Gamma\left(1+5 e^{-w_{1} \zeta}\right)}\right) \\
& \quad+10 w_{1}^{2}\left(1-w_{1}\right)^{2}
\end{aligned}
$$

Subjects to the IC:

$$
\Psi\left(w_{1}, 0\right)=10 w_{1}^{2}\left(1-w_{1}\right)^{2}, \quad 0 \leq w_{1} \leq 1
$$

And Dirichlet BC:

$$
\begin{aligned}
& \Psi(0, \zeta)=0 \quad 0 \leq \zeta \leq J \\
& \Psi(1, \zeta)=0
\end{aligned}
$$

That the exact solution to this problem is:

$$
\Psi\left(w_{1}, \zeta\right)=10 w_{1}^{2}\left(1-w_{1}\right)^{2}(1+\zeta), \quad 0 \leq w_{1} \leq 1 .
$$

Table 1 displays the proposed method obtained for $\mathrm{J}=1$ and comparison between error our method and $\frac{45-46}{}$. Figures 1-2 show the plot of the numerical and the exact solution surface respectively. Table 2 presents comparison between the absolute value of the maximum errors (MEs) of proposed method and 5 -46.

Example 2: Consider the problem (1) with the following $\mathrm{BC}$ and IC:

$$
\begin{aligned}
& \beta_{1} D_{\zeta} \Psi\left(w_{1}, \zeta\right)+\beta_{2} D_{\zeta}^{0.8+0.005 \cos \left(w_{1} \zeta\right) \sin \left(w_{1} \zeta\right)} \Psi\left(w_{1}, \zeta\right) \\
& =-\beta_{3} D_{w_{1}} \Psi\left(w_{1}, \zeta\right)+\beta_{4} D_{w_{1}}^{2} \Psi\left(w_{1}, \zeta\right)+Q\left(w_{1}, \zeta\right)
\end{aligned}
$$


Table 1. Numerical solution, exact solution and absolute error at $J=1$

\begin{tabular}{|l|l|l|l|l|l|}
\hline$W_{1}$ & proposed method & Exact Solution & Error of $\underline{45}$ & Error of $\underline{46}$ & Error of proposed method \\
\hline 0.1 & 0.162 & 0.162 & $1.56290 \times 10^{-4}$ & $4.77812 \times 10^{-15}$ & 0.000 \\
\hline 0.2 & 0.512 & 0.512 & $1.40069 \times 10^{-3}$ & $9.13997 \times 10^{-16}$ & 0.000 \\
\hline 0.3 & 0.882 & 0.882 & $2.97519 \times 10^{-3}$ & $2.76073 \times 10^{-16}$ & 0.000 \\
\hline 0.4 & 1.152 & 1.152 & $4.29766 \times 10^{-3}$ & $7.07249 \times 10^{-16}$ & 0.000 \\
\hline 0.5 & 1.250 & 1.250 & $4.97219 \times 10^{-3}$ & $1.04861 \times 10^{-17}$ & 0.000 \\
\hline 0.6 & 1.152 & 1.152 & $4.80341 \times 10^{-3}$ & $2.33975 \times 10^{-16}$ & 0.000 \\
\hline 0.7 & 0.882 & 0.882 & $3.81527 \times 10^{-3}$ & $2.30960 \times 10^{-16}$ & 0.000 \\
\hline 0.8 & 0.512 & 0.512 & $2.27469 \times 10^{-3}$ & $8.43242 \times 10^{-17}$ & 0.000 \\
\hline 0.9 & 0.162 & 0.162 & $7.20750 \times 10^{-4}$ & $7.95279 \times 10^{-17}$ & 0.000 \\
\hline
\end{tabular}

Table 2. Comparison based on MEs of our method ${ }^{45}$

\begin{tabular}{|l|l|l|}
\hline$h=\tau$ & ME of ${ }^{45}$ & ME of our method \\
\hline $1 / 50$ & $9.4391 \times 10^{-3}$ & 0.0000 \\
\hline $1 / 100$ & $5.0134 \times 10^{-3}$ & 0.0000 \\
\hline $1 / 200$ & $2.5613 \times 10^{-3}$ & 0.0000 \\
\hline $1 / 400$ & $1.2781 \times 10^{-3}$ & 0.0000 \\
\hline
\end{tabular}

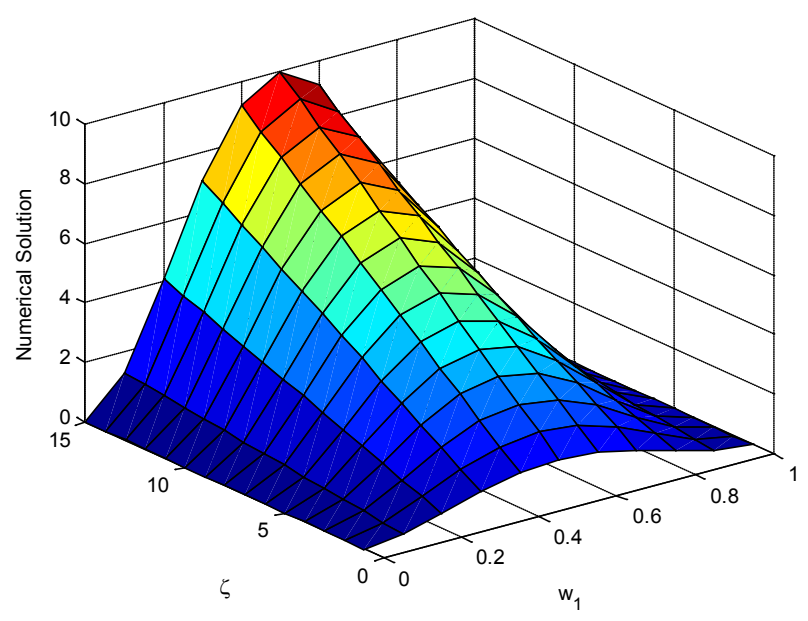

Figure 1. The surfaces show the numerical solutions $\Psi\left(w_{1}, \zeta\right)$ of eq. (7).

Let $\beta_{1}=\beta_{2}=\beta_{3}=\beta_{4}=1$

And the source functions:

$$
\begin{aligned}
& Q\left(w_{1}, \zeta\right)=(5 \zeta+5)\left(1-w_{1}\right)-(5 \zeta+5) w_{1}-2(-10 \zeta-10) \\
& -5 w_{1}\left(1-w_{1}\right)+5 w_{1}\left(1-w_{1}\right)\left(\frac{\zeta^{0.2-0.5 e-2 \cos \left(w_{1} \zeta\right) \sin \left(w_{1}\right)}}{\Gamma\left(2-\zeta^{0.2-0.5 e-2 \cos \left(w_{1} \zeta\right) \sin \left(w_{1}\right)}\right)}\right)
\end{aligned}
$$

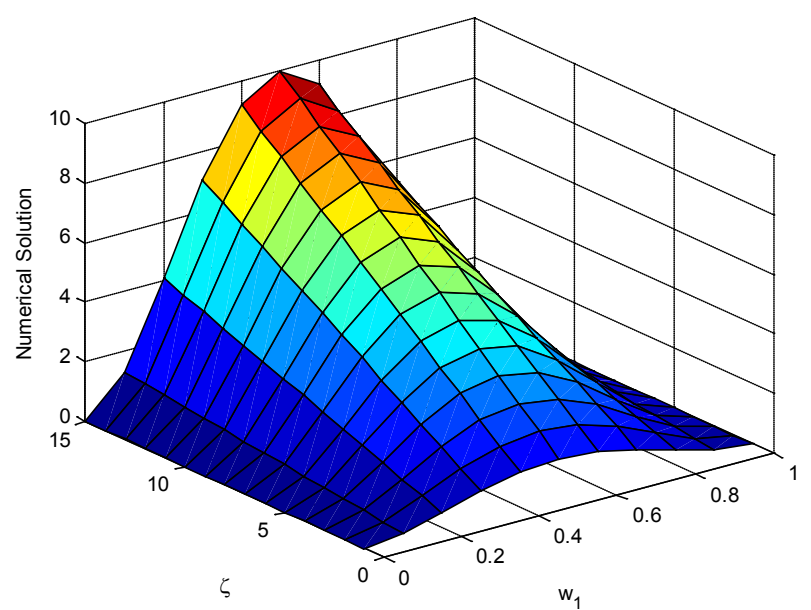

Figure 2. The surfaces show the exact solution $\Psi\left(w_{1}, \zeta\right)$ of eq. (7).

Subjects to the IC:

$\Psi\left(w_{1}, 0\right)=5 w_{1}\left(1-w_{1}\right), \quad 0 \leq w_{1} \leq 1$

And Dirichlet BC:

$$
\begin{aligned}
& \Psi(0, \zeta)=0 \\
& \Psi(1, \zeta)=0
\end{aligned}
$$

That the exact solution to this problem is:

$$
\Psi\left(w_{1}, \zeta\right)=5 w_{1}\left(1-w_{1}\right)(\zeta+1), 0 \leq w_{1} \leq 1 .
$$


Table 3 presents comparison between the absolute value of the MEs of our method and ${ }^{45}$. Figures 3-4 show the plot of the numerical and the exact solution surface respectively. Table 4 shows some of the analytical solutions for VFOMAD M, obtained for different values and comparison between exact solution and analytical solution.

Example 3: Consider the problem (1) with the following IC and BC:

$$
\begin{aligned}
& \beta_{1} D_{\zeta} \Psi\left(w_{1}, w_{2}, \zeta\right)+\beta_{2} D_{\zeta}^{1-0.5 e^{-w_{1} w_{2} \zeta}} \Psi\left(w_{1}, w_{2}, \zeta\right)= \\
& -\beta_{3} D_{w_{1}} \Psi\left(w_{1}, w_{2}, \zeta\right)-\beta_{3} D_{w_{2}} \Psi\left(w_{1}, w_{2}, \zeta\right) \\
& +\beta_{4} D_{w_{1}}^{2} \Psi\left(w_{1}, w_{2}, \zeta\right)+\beta_{4} D_{w_{2}}^{2} \Psi\left(w_{1}, w_{2}, \zeta\right) \\
& +Q\left(w_{1}, w_{2}, \zeta\right)
\end{aligned}
$$

Let $\beta_{1}=\beta_{2}=\beta_{3}=\beta_{4}=1$

Table 3. Comparison based on MEs of our method and ${ }^{45}$ at $J=1$

\begin{tabular}{|l|l|l|}
\hline$h=\tau$ & MEs of $\underline{48}$ & MEs of our method \\
\hline $1 / 50$ & $2.1562 \times 10^{-2}$ & 0.0000 \\
\hline $1 / 100$ & $1.0825 \times 10^{-2}$ & 0.0000 \\
\hline $1 / 200$ & $5.4267 \times 10^{-3}$ & 0.0000 \\
\hline $1 / 400$ & $2.7164 \times 10^{-3}$ & 0.0000 \\
\hline
\end{tabular}

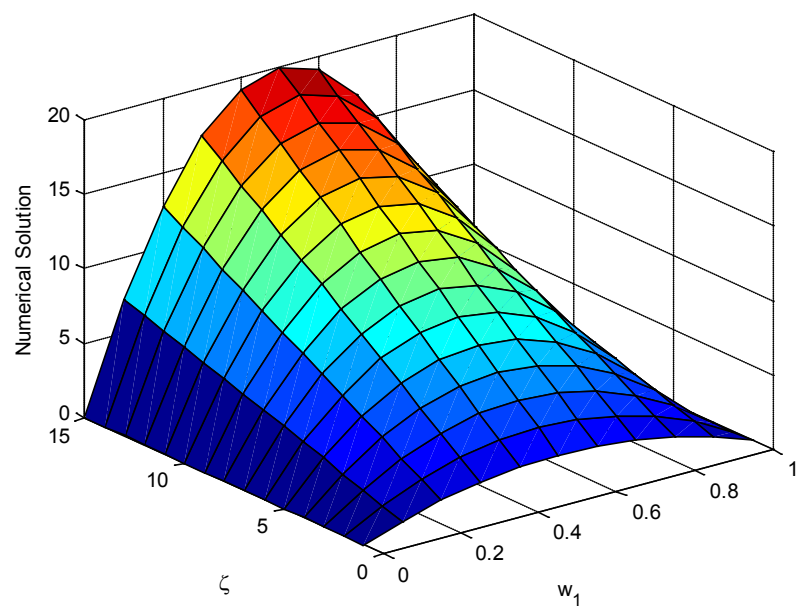

Figure 3. The surfaces show the Numerical solution $\Psi\left(w_{1}, \zeta\right)$ of eq. (8).

\begin{tabular}{|c|c|c|c|c|}
\hline$W_{1}$ & $\zeta$ & $\begin{array}{l}\text { Exact } \\
\text { Solution }\end{array}$ & $\begin{array}{l}\text { Variational } \\
\text { Iteration Method }\end{array}$ & |uex-uVIM| \\
\hline 0 & 0.2 & 0.000 & 0.000 & 0.000 \\
\hline 0.1 & 0.2 & 0.540 & 0.540 & 0.000 \\
\hline 0.2 & 0.2 & 0.960 & 0.960 & 0.000 \\
\hline 0.3 & 0.2 & 1.260 & 1.260 & 0.000 \\
\hline 0.4 & 0.2 & 1.440 & 1.440 & 0.000 \\
\hline 0.5 & 0.2 & 1.500 & 1.500 & 0.000 \\
\hline 0.6 & 0.2 & 1.440 & 1.440 & 0.000 \\
\hline 0.7 & 0.2 & 1.260 & 1.260 & 0.000 \\
\hline 0.8 & 0.2 & 0.960 & 0.960 & 0.000 \\
\hline 0.9 & 0.2 & 0.540 & 0.540 & 0.000 \\
\hline 1 & 0.2 & 0.000 & 0.000 & 0.000 \\
\hline 0 & 0.3 & 0.000 & 0.000 & 0.000 \\
\hline 0.1 & 0.3 & 0.585 & 0.585 & 0.000 \\
\hline 0.2 & 0.3 & 1.040 & 1.040 & 0.000 \\
\hline 0.3 & 0.3 & 1.365 & 1.365 & 0.000 \\
\hline 0.4 & 0.3 & 1.560 & 1.560 & 0.000 \\
\hline 0.5 & 0.3 & 1.620 & 1.620 & 0.000 \\
\hline 0.6 & 0.3 & 1.560 & 1.560 & 0.000 \\
\hline 0.7 & 0.3 & 4.200 & 4.200 & 0.000 \\
\hline 0.8 & 0.3 & 3.200 & 3.200 & 0.000 \\
\hline 0.9 & 0.3 & 1.800 & 1.800 & 0.000 \\
\hline 1 & 0.3 & 0.000 & 0.000 & 0.000 \\
\hline 0 & 0.4 & 0.000 & 0.000 & 0.000 \\
\hline 0.1 & 0.4 & 0.630 & 0.630 & 0.000 \\
\hline 0.2 & 0.4 & 1.120 & 1.120 & 0.000 \\
\hline 0.3 & 0.4 & 1.470 & 1.470 & 0.000 \\
\hline 0.4 & 0.4 & 1.680 & 1.680 & 0.000 \\
\hline 0.5 & 0.4 & 1.750 & 1.750 & 0.000 \\
\hline 0.6 & 0.4 & 1.680 & 1.680 & 0.000 \\
\hline 0.7 & 0.4 & 1.470 & 1.470 & 0.000 \\
\hline 0.8 & 0.4 & 1.120 & 1.120 & 0.000 \\
\hline 0.9 & 0.4 & 0.630 & 0.630 & 0.000 \\
\hline 1 & 0.4 & 0.000 & 0.000 & 0.000 \\
\hline
\end{tabular}

Table 4. Some of comparison between exact solution and analytical solution when $\alpha\left(w_{1}, \zeta\right)=0.8+0.005 \cos \left(w_{1} \zeta\right) \sin \left(w_{1} \zeta\right)$ for example 2 


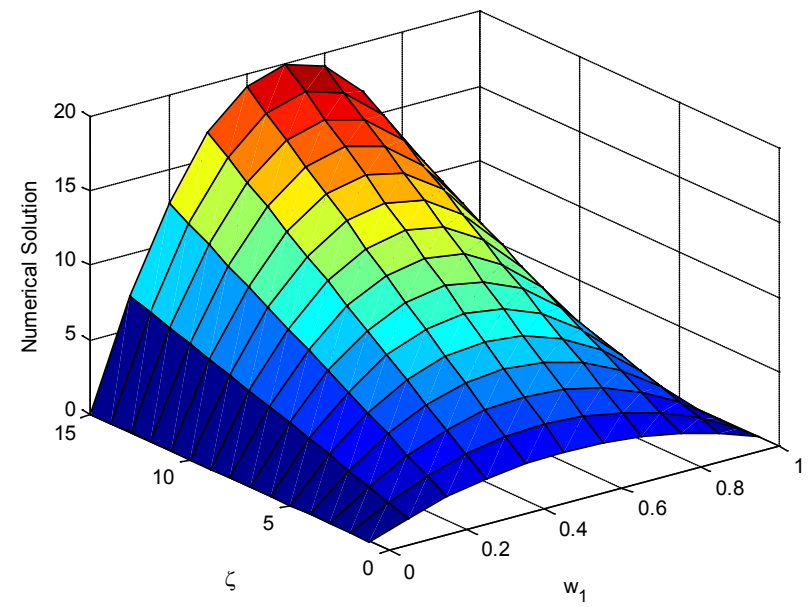

Figure 4. The surfaces show the exact solution $\Psi\left(w_{1}, \zeta\right)$ of eq. (8)

And the source functions:

$$
\begin{aligned}
& Q\left(w_{1}, w_{2}, \zeta\right)=2 w_{1}, w_{2}^{2}(10 \zeta+10)\left(1-w_{1}\right)^{2}\left(1-w_{2}\right)^{2} \\
& -2 w_{1}^{2} w_{2}^{2}(10 \zeta+10)\left(1-w_{1}\right)\left(1-w_{2}\right)^{2} \\
& \quad-2 w_{2}^{2}(10+10)\left(1-w_{1}\right)^{2}\left(1-w_{2}\right)^{2} \\
& \quad-8 w_{1} w_{2}^{2}(10 \zeta+10)\left(1-w_{1}\right)\left(1-w_{2}\right)^{2} \\
& +2 w_{1}^{2} w_{2}^{2}(10 \zeta+10)\left(1-w_{2}\right)^{2} \\
& +2 w_{1}^{2} w_{2}(10 \zeta+10)\left(1-w_{1}\right)^{2}\left(1-w_{2}\right)^{2} \\
& -2 w_{1}^{2} w_{2}^{2}(10 \zeta+10)\left(1-w_{1}\right)^{2}\left(1-w_{2}\right) \\
& +2 w_{1}^{2}(10 \zeta+10)\left(1-w_{1}\right)^{2}\left(1-w_{2}\right)^{2} \\
& +2 w_{1}^{2} w_{2}(10 \zeta+10)\left(1-w_{1}\right)^{2}\left(1-w_{2}\right) \\
& +2 w_{1}^{2} w_{2}^{2}(10 \zeta+10)\left(1-w_{1}\right)^{2} \\
& +10 w_{1}^{2} w_{2}^{2}\left(1-w_{1}\right)^{2}\left(1-w_{2}\right)^{2} \\
& +10 w_{1}^{2} w_{2}^{2}\left(1-w_{1}\right)^{2}\left(1-w_{2}\right)^{2}\left(\frac{\zeta^{5 e^{-w_{1} w_{2} \zeta}}}{\Gamma\left(1+5 e^{-w_{1} w_{2} \zeta}\right)}\right)
\end{aligned}
$$

Subjects to the IC:

\begin{tabular}{|c|c|c|c|c|}
\hline $\boldsymbol{w}_{1}=\boldsymbol{w}_{2}$ & $\zeta$ & $\begin{array}{l}\text { Exact } \\
\text { Solution }\end{array}$ & $\begin{array}{l}\text { Variational } \\
\text { Iteration Method }\end{array}$ & $\mid \Psi_{e x}-\Psi_{V I M}$ \\
\hline 0 & 1 & 0.000 & 0.000 & 0.000 \\
\hline 0.1 & 1 & $1.312 \times 10^{-3}$ & $1.312 \times 10^{-3}$ & 0.000 \\
\hline 0.2 & 1 & 0.013 & 0.013 & 0.000 \\
\hline 0.3 & 1 & 0.039 & 0.039 & 0.000 \\
\hline 0.4 & 1 & 0.066 & 0.066 & 0.000 \\
\hline 0.5 & 1 & 0.078 & 0.078 & 0.000 \\
\hline 0.6 & 1 & 0.066 & 0.066 & 0.000 \\
\hline 0.7 & 1 & 0.039 & 0.039 & 0.000 \\
\hline 0.8 & 1 & 0.013 & 0.013 & 0.000 \\
\hline 0.9 & 1 & $1.312 \times 10^{-3}$ & $1.312 \times 10^{-3}$ & 0.000 \\
\hline 1 & 1 & 0.000 & 0.000 & 0.000 \\
\hline 0 & 2 & 0.000 & 0.000 & 0.000 \\
\hline 0.1 & 2 & $1.968 \times 10^{-3}$ & $1.968 \times 10^{-3}$ & 0.000 \\
\hline 0.2 & 2 & 0.020 & 0.020 & 0.000 \\
\hline 0.3 & 2 & 0.058 & 0.058 & 0.000 \\
\hline 0.4 & 2 & 0.100 & 0.100 & 0.000 \\
\hline 0.5 & 2 & 0.117 & 0.117 & 0.000 \\
\hline 0.6 & 2 & 0.100 & 0.100 & 0.000 \\
\hline 0.7 & 2 & 0.058 & 0.058 & 0.000 \\
\hline 0.8 & 2 & 0.020 & 0.020 & 0.000 \\
\hline 0.9 & 2 & $1.968 \times 10^{-3}$ & $1.968 \times 10^{-3}$ & 0.000 \\
\hline 1 & 2 & 0.000 & 0.000 & 0.000 \\
\hline 0 & 3 & 0.000 & 0.000 & 0.000 \\
\hline 0.1 & 3 & $2.624 \times 10^{-3}$ & $2.624 \times 10^{-3}$ & 0.000 \\
\hline 0.2 & 3 & 0.026 & 0.026 & 0.000 \\
\hline 0.3 & 3 & 0.078 & 0.078 & 0.000 \\
\hline 0.4 & 3 & 0.133 & 0.133 & 0.000 \\
\hline 0.5 & 3 & 0.156 & 0.156 & 0.000 \\
\hline 0.6 & 3 & 0.133 & 0.133 & 0.000 \\
\hline 0.7 & 3 & 0.078 & 0.078 & 0.000 \\
\hline 0.8 & 3 & 0.026 & 0.026 & 0.000 \\
\hline 0.9 & 3 & $2.624 \times 10^{-3}$ & $2.624 \times 10^{-3}$ & 0.000 \\
\hline 1 & 3 & 0.000 & 0.000 & 0.000 \\
\hline
\end{tabular}

$\Psi\left(w_{1}, w_{2}, 0\right)=10 w_{1}^{2} w_{2}^{2}\left(1-w_{1}\right)^{2}\left(1-w_{2}\right)^{2}, \quad 0 \leq w_{1}, w_{2} \leq 1$

And Dirichlet BC:

$$
\begin{aligned}
& \Psi\left(0, w_{2}, \zeta\right)=0 \\
& \Psi\left(w_{1}, 0, \zeta\right)=0 \\
& \Psi\left(1, w_{2}, \zeta\right)=0 \\
& \Psi\left(w_{1}, 1, \zeta\right)=0
\end{aligned}
$$

That the exact solution to this problem is:

$$
\begin{aligned}
& \Psi\left(w_{1}, w_{2}, \zeta\right)=10 w_{1}^{2} w_{2}^{2}\left(1-w_{1}\right)^{2}\left(1-w_{2}\right)^{2} \\
& (1+\zeta), 0 \leq w_{1}, w_{2} \leq 1 .
\end{aligned}
$$

Table 5 shows part the analytical solutions for VFOMADM with 2-D obtained for different values and comparison between exact solution and analytical solution. Table 6

Table 5. Some of comparison between exact solution and analytical solution when $\alpha\left(w_{1}, w_{2}, \zeta\right)=1-0.5 e^{-w_{1} w_{2} \zeta}$ for example 3 
Table 6. MEs of the numerical solution at $J=1,2,3$

\begin{tabular}{|l|l|}
\hline$h=\tau$ & MEs of our method \\
\hline $1 / 50$ & 0.0000 \\
\hline $1 / 100$ & 0.0000 \\
\hline $1 / 200$ & 0.0000 \\
\hline $1 / 400$ & 0.0000 \\
\hline
\end{tabular}

presents the absolute value of the MEs of the numerical solution at $J=1,2,3$.

Example 4: Consider the problem (1) with the following $\mathrm{BC}$ and IC:

$$
\begin{aligned}
& \beta_{1} D_{\zeta} \Psi\left(w_{1}, w_{2}, \zeta\right)+\beta_{2} D_{\zeta}^{0.8+0.005 \cos \left(w_{1} w_{2} \zeta\right) \sin \left(w_{1} w_{2}\right)} \Psi\left(w_{1}, w_{2}, \zeta\right)= \\
& -\beta_{3} D_{w_{1}} \Psi\left(w_{1}, w_{2}, \zeta\right)-\beta_{3} D_{w_{2}} \Psi\left(w_{1}, w_{2}, \zeta\right) \\
& +\beta_{4} D_{w_{1}}^{2} \Psi\left(w_{1}, w_{2}, \zeta\right)+\beta_{4} D_{w_{2}}^{2} \Psi\left(w_{1}, w_{2}, \zeta\right) \\
& +Q\left(w_{1}, w_{2}, \zeta\right)
\end{aligned}
$$

Let $\beta_{1}=\beta_{2}=\beta_{3}=\beta_{4}=1$

And the source functions:

$$
\begin{aligned}
& Q\left(w_{1}, w_{2}, \zeta\right)=2 w_{1} w_{2}(5 \zeta+5)\left(1-w_{1}\right)\left(1-w_{2}\right) \\
& \quad-w_{1}^{2} w_{2}(5 \zeta+5)\left(1-w_{2}\right) \\
& \quad+w_{1}^{2}(5 \zeta+5)\left(1-w_{1}\right)\left(1-w_{2}\right) \\
& \quad-w_{1}^{2} w_{2}(5 \zeta+5)\left(1-w_{1}\right)-2 w_{2}(5 \zeta+5) \\
& \quad\left(1-w_{1}\right)\left(1-w_{2}\right)+4 w_{1} w_{2}(5 \zeta+5)\left(1-w_{2}\right) \\
& \quad+2 w_{1}^{2}(5 \zeta+5)\left(1-w_{1}\right)+5 w_{1}^{2} w_{2}\left(1-w_{1}\right)\left(1-w_{2}\right) \\
& \quad+5 w_{1} w_{2}\left(1-w_{1}\right)\left(1-w_{2}\right)\left(\frac{\zeta^{0.2-0.5 e-2 \cos \left(w_{1} w_{2} \zeta\right) \sin \left(w_{1} w_{2}\right)}}{\Gamma\left(2-\zeta^{0.2-0.5 e-2 \cos \left(w_{1} w_{2} \zeta\right) \sin \left(w_{1} w_{2}\right)}\right)}\right)
\end{aligned}
$$

Subjects to the IC:

$\Psi\left(w_{1}, w_{2}, 0\right)=5 w_{1}\left(1-w_{1}\right)\left(1-w_{2}\right), \quad 0 \leq w_{1}, w_{2} \leq 1$

And Dirichlet BC:

$$
\begin{aligned}
& \Psi\left(0, w_{2}, \zeta\right)=0 \\
& \Psi\left(w_{1}, 0, \zeta\right)=0 \\
& \Psi\left(1, w_{2}, \zeta\right)=0 \\
& \Psi\left(w_{1}, 1, \zeta\right)=0
\end{aligned}
$$

That the exact solution to this problem is:

$$
\begin{aligned}
& \Psi\left(w_{1}, w_{2}, \zeta\right)=5 w_{1} w_{2}\left(1-w_{1}\right)\left(1-w_{2}\right)(\zeta+1), \\
& 0 \leq w_{1}, w_{2} \leq 1
\end{aligned}
$$

Table 7 shows part the analytical solutions for VFOMADM with 2-D obtained for different values and comparison between exact solution and analytical solution. Table 8 presents the absolute value of the MEs of the numerical solution.

Table 7. Some of comparison between exact solution and analytical solution when $\alpha\left(w_{1}, w_{2}, \zeta\right)=0.8+0.005 \cos \left(w_{1} w_{2} \zeta\right) \sin \left(w_{1} w_{2}\right)$ for example 4

\begin{tabular}{|l|l|l|l|l|}
\hline $\boldsymbol{w}_{1}=\boldsymbol{w}_{2}$ & $\zeta$ & $\begin{array}{l}\text { Exact } \\
\text { Solution }\end{array}$ & $\begin{array}{l}\text { Variational } \\
\text { Iteration } \\
\text { Method }\end{array}$ & $\left|\Psi_{\text {ex }}-\Psi_{\text {VIM }}\right|$ \\
\hline 0 & 3 & 0.00000 & 0.00000 & 0.000 \\
\hline 0.1 & 3 & 0.01600 & 0.01600 & 0.000 \\
\hline 0.2 & 3 & 0.10240 & 0.10240 & 0.000 \\
\hline 0.3 & 3 & 0.26460 & 0.26460 & 0.000 \\
\hline 0.4 & 3 & 0.46080 & 0.46080 & 0.000 \\
\hline 0.5 & 3 & 0.62500 & 0.62500 & 0.000 \\
\hline 0.6 & 3 & 0.69120 & 0.69120 & 0.000 \\
\hline 0.7 & 3 & 0.61740 & 0.61740 & 0.000 \\
\hline 0.8 & 3 & 0.40960 & 0.40960 & 0.000 \\
\hline 0.9 & 3 & 0.14600 & 0.14600 & 0.000 \\
\hline 1 & 3 & 0.00000 & 0.00000 & 0.000 \\
\hline 0 & 4 & 0.00000 & 0.00000 & 0.000 \\
\hline 0.1 & 4 & 0.02000 & 0.02000 & 0.000 \\
\hline 0.2 & 4 & 0.12800 & 0.12800 & 0.000 \\
\hline 0.3 & 4 & 0.33075 & 0.33075 & 0.000 \\
\hline 0.4 & 4 & 0.57600 & 0.57600 & 0.000 \\
\hline 0.5 & 4 & 0.78125 & 0.78125 & 0.000 \\
\hline 0.6 & 4 & 0.86400 & 0.86400 & 0.000 \\
\hline 0.7 & 4 & 0.77175 & 0.77175 & 0.000 \\
\hline 0.8 & 4 & 0.51200 & 0.51200 & 0.000 \\
\hline 0.9 & 4 & 0.18200 & 0.18200 & 0.000 \\
\hline 1 & 4 & 0.00000 & 0.00000 & 0.000 \\
\hline & & & & \\
\hline & & & \\
\hline & & & \\
\hline
\end{tabular}

Table 8. MEs of the numerical solution at $J=1,2,3$

\begin{tabular}{|l|l|}
\hline$h=\tau$ & MEs of our method \\
\hline 0.200 & 0.0000 \\
\hline 0.100 & 0.0000 \\
\hline 0.050 & 0.0000 \\
\hline 0.025 & 0.0000 \\
\hline
\end{tabular}




\section{Conclusion}

In this paper, the results obtained for modified treatment of initial boundary conditions for the MDMADEVFwere accurate. This algorithm was simple and easy to implement. The numerical results demonstrated that the method was accurate. On the other hand, reliable and converges faster with less computation when compared with other methods in the literature.

\section{References}

1. Garrappa R, Popolizio M. On the use of matrix functions for fractional partial differential equations. Mathematics and Computers in Simulation. 2011; 81(5):1045-56. https://doi.org/10.1016/j.matcom.2010.10.009

2. Li C, Deng W. Remarks on fractional derivatives. Applied Mathematics and Computation. 2007; 187(2):777-84. https://doi.org/10.1016/j.amc.2006.08.163

3. Hilfer R. Applications of Fractional Calculus in Physics. Word Scientific Publishing Co., New Jersey, London, Hong Kong. 2000. https://doi.org/10.1142/3779 PMid:11088546

4. Podlubny I. Fractional Differential Equations. Academic Press Inc, San Diego, CA. 1999.

5. Magin L. Fractional Calculus in Bioengineering. Begell House Publishers. 2006.

6. Kirchner W, Feng X, Neal C. Fractal stream chemistry and its implications for contaminant transport in catchments. Nature. 2000; 403(6769): 524-7. https://doi. org/10.1038/35000537 PMid:10676956

7. Giona M, Roman E. Fractional diffusion equation and relaxation in complex viscoelastic materials. Physica A: Statistical Mechanics and its Applications. 1992; 191:449-53. https://doi.org/10.1016/0378-4371(92)90566-9

8. MeerschaertM, Tadjeran C. Finite difference approximations for two-sided space-fractional partial differential equations. Applied Numerical Mathematics. 2006; 56(1): 80-90. https://doi.org/10.1016/j.apnum.2005.02.008

9. Ding Z, Xiao A, Li M. Weighted finite difference methods for a class of space fractional partial differential equations with variable coefficients. Journal of Computational and Applied Mathematics. 2010; 233(8):1905-14. https://doi.org/10.1016/j.cam.2009.09.027

10. Wang H, Du N. Fast Alternating-Direction Finite Difference Methods for Three-Dimensional Space-Fractional Diffusion Equations. Journal of Computational Physics. 2014; 258: 305-18. https://doi.org/10.1016/j.jcp.2013.10.040

11. Ma J, Liu J, Zhou Z. Convergence analysis of moving finite element methods for space fractional differential equations. Journal of Computational and Applied Mathematics. 2014; 255: 661-70. https://doi.org/10.1016/j.cam.2013.06.021
12. Jiang Y, Ma J. High-order finite element methods for time-fractional partial differential equations. Journal of Computational and Applied Mathematics. 2011; 235(11):3285-90.https://doi.org/10.1016/j.cam.2011.01.011

13. Zhang H, Liu F, Anh V. Garlerkin finite element approximations of symmetric space-fractional partial differential equations. Applied Mathematics and Computation. 2010; 217 (6):2534-45. https://doi.org/10.1016/j.amc.2010.07.066

14. Li L, Xu D, Luo M. Alternating direction implicit galerkinfinite element method for the two-dimensional fractional diffusion-wave equation. Journal of Computational Physics. 2013; 255(1):471-85. https://doi.org/10.1016/j. jcp.2013.08.031

15. Bhrawy H, Alghamdi A. A shifted Jacobi-Gauss-Lobatto collocation method for solving nonlinear fractional Langevin equation involving two fractional orders in different intervals. Boundary value problems. 2010; 2012:1-62.

16. Bhrawy H, Alofi S. The operational matrix of fractional integration for shifted Chebyshev polynomials. Applied Mathematics Letters. 2013; 26 (1): 25-31. https://doi. org/10.1016/j.aml.2012.01.027

17. Bhrawy H, Baleanu D. A Spectral Legendre-GaussLobattocollocation method for a space fractional advection diffusion equation with variable coefficients. Reports on Mathematical Physics. 2013; 72(2):219-33. https://doi.org/10.1016/S0034-4877(14)60015-X

18. Bhrawy H, Alhamed A, Baleanu D, Al-Zahrani A. New special techniques for systems of fractional differential equations using fractional-order generalized Laguerre orthogonal functions. Fractional Calculus and Applied Analysis. 2014; 17 (4): 1137-57. https://doi.org/10.2478/ s13540-014-0218-9

19. Bhrawy H, Doha H, Baleanu D, Ezz-Eldien S. A spectral tau algorithm based on Jacobi operational matrix for numerical solution of time fractional diffusion-wave equations. Jornal of Computational Physics. 2015; 293:142-56 https://doi.org/10.1016/j.jcp.2014.03.039

20. Meerschaert M, Benson J, Baumer B. Multidimensional advection and fractional dispersion. Physical Review. 1999; 59 (5): 5026-8. https://doi.org/10.1103/PhysRevE.59.5026

21. Cooper J, Cowan R. Filtering using variable order vertical derivatives.ComputersandGeosciences. 2004;30(5):455-59. https://doi.org/10.1016/j.cageo.2004.03.001

22. Tseng C. Design of variable and adaptive fractional order FIR differentiators. Signal Processing. 2006; 86 (10): 2554-66. https://doi.org/10.1016/j.sigpro.2006.02.004

23. Sun $H$, Chen W, Chen Y. Variable-order fractional differential operators in anomalous diffusion modeling. Physica A: Statistical Mechanics and its Applications. 2009; 388 (21): pp. 4586-92. https://doi.org/10.1016/j.physa.2009.07.024 
24. Samko, G, Ross, B. Integration and differentiation to a variable fractional order. Integral Transforms and Special Functions. 1993; 1(4): 277-300. https://doi. org/10.1080/10652469308819027

25. Ross B, Samko G. Fractional integration operator of variable order in the HSlder spaces $\mathrm{H} \lambda(\mathrm{x})$. International Journal of MathematicsandMathematicalSciences.1995;18(4):777-88. https://doi.org/10.1155/S0161171295001001

26. Samko G. Fractional integration and differentiation of variable order. Analysis Mathematica. 1995; 21(3): 213-36. https://doi.org/10.1007/BF01911126

27. LorenzoF,HartleyT.Variableorderanddistributedorderfractional operators. Nonlinear dynamics. 2002; 29 (1-4): 57-98 https://doi.org/10.1023/A:1016586905654

28. Ingman D, Suzdalnitsky J. Computer Methods in Applied Mechanics and Engineering. 2004; 193: 5585-95. https://doi.org/10.1016/j.cma.2004.06.029

29. Pedro C, Kobayashi H, Pereira C, Coimbra M. Variable Order Modeling of Diffusive Convective Effects on The Oscillatory Flow Past A Sphere. Journal of Vibration and Control. 2008; 14:1659-72. https://doi.org/10.1177/1077546307087397

30. Ramirez S, Coimbra M. On the selection and meaning of variable order operators for dynamic modeling. International Journal of Differential equations. 2010; 1-16. https://doi.org/10.1155/2010/846107

31. Lin R, Liu F, Anh V, Turner I. Stability and convergence of a new explicit finite-difference approximation for the variable-order nonlinear fractional diffusion equation. Applied Mathematics and Computation. 2009; 212 (2): 435-45. https://doi.org/10.1016/j.amc.2009.02.047

32. Zhuang P, Liu F, Anh V, Turner I. Numerical Methods for the Variable-Order Fractional Advection-Diffusion Equation with a Nonlinear Source Term. SIAM Journal on Numerical Analysis. 2009; 47 (3): 1760-81. https://doi. org/10.1137/080730597

33. Chen C, Liu F, Anh V, Turner I. Numerical schemes with high spatial accuracy for a variable-order anomalous subdiffusion equation. SIAM Journal on Scientific Computing. 2010; 32 (4): 1740-60. https://doi.org/10.1137/090771715

34. Chen M, Wei Q, Liu Y, Yu H. Numerical solution for a class of nonlinear variable order fractional differential equations with Legendre wavelets. Applied Mathematics Letters. 2015; 46: 83-8. https://doi.org/10.1016/j.aml.2015.02.010

35. Shen S, Liu F, Chen J, Turner I, Anh V. Numerical techniques for the variable order time fractional diffusion equation. Journal of Applied Mathematics and Computing. 2012; 218 (22):10861-70. https://doi.org/10.1016/j.amc.2012.04.047

36. Shen S, Liu F, AnhV, Turner I, Chen J. A characteristic difference method for the variable-order fractional advection-diffusion equation. Journal of Applied
Mathematics and Computing. 2013; 42 (1-2): 371-86. https://doi.org/10.1007/s12190-012-0642-0

37. Zhao X, Sun Z, Karniadakis E. Second-order approximations for variable order fractional derivatives: algorithms and applications. Journal of Computational Physics. 2015; 293:184-200. https://doi.org/10.1016/j.jcp.2014.08.015

38. Bhrawy H. A highly accurate collocation algorithm for $1+1$ and $2+1$ fractional percolation equations. Journal of Vibration and Control. 2016; 22 (9): 2288-310. https://doi. org/10.1177/1077546315597815

39. Bhrawy H, Abdelkawy A. A fully spectral collocation approximation for multi-dimensional fractional Schrodinger equations. Journal of Computational Physics. 2015; 294:462-83. https://doi.org/10.1016/j.jcp.2015.03.063

40. 40.Bhrawy H, Zaky A, Machado T. Efficient Legendre spectral tau algorithmfor solving two-sided spacetime Caputo fractional advection-dispersion equation. Journal of Vibration and Control. 2015; 22 (8): 2053-68. https://doi.org/10.1177/1077546314566835

41. Bhrawy H. A Jacobi spectral collocation method for solving multidimensional nonlinear fractional sub diffusion equations. Numerical Algorithms. 2016; 73:91-113. https://doi.org/10.1007/s11075-015-0087-2

42. Bhrawy H, Zaky A. Numerical simulation for twodimensional variable-order fractional nonlinear cable equation. Nonlinear Dynamics. 2015; 80 (1-2): 101-16. https://doi.org/10.1007/s11071-014-1854-7

43. Zayernouri M, Karniadakis E. Fractional spectral collocation methods for linear and nonlinear variable order FPDEs. Journal of Computational Physics. 2015; 293: 312-38. https://doi.org/10.1016/j.jcp.2014.12.001

44. Li Y, Wu Y. A numerical technique for variable fractional functional boundary value problems. Applied Mathematics Letters. 2015; 43: 108-13. https://doi.org/10.1097/01. ccm.0000473871.99911.fa

45. Zhang H, Liu F, Phanikumar S, Meerschaert M. A novel numerical method for the time variable fractional order mobile-immobile advection-dispersion model. Computers and Mathematics with Applications. 2013; 66 (5): 693-701. https://doi.org/10.1016/j.camwa.2013.01.031

46. Abdelkawy A, Mahmoud Z, Bhrawy A, Baleanu D. Numerical simulation of time variable fractional order mobile-immobile advection-dispersion model. Romanian Reports in Physics. 2016; 67 (3): 773-91.

47. Chen Y, Liu L, Li B, Sun Y. Numerical solution for the variable order linear cable equation with Bernstein polynomials. Applied Mathematics and Computation. 2014; 238: 329-41. https://doi.org/10.1016/j.amc.2014.03.066

48. Manohar M. Matrix method for numerical solution of space-time fractional diffusion-wave equations with three space variables. Afrika Matematika. 2014; 25 (1): 161-81. https://doi.org/10.1007/s13370-012-0101-y 